\title{
CHARACTER SEMIGROUPS OF LOCALLY COMPACT INVERSE SEMIGROUPS
}

\author{
RONALD O. FULP
}

\begin{abstract}
We show that if $S$ is a locally compact abelian continuous-inverse semigroup whose idempotent semigroup $E$ satisfies a certain technical condition on its prime ideals, then the maximal subgroups of the character semigroup $S^{\wedge}$ of $S$ are obtained as inverse limits of the duals of the maximal subgroups of $S$. It is shown that the technical conditions on $E$ are satisfied in each of the following cases: $E$ is compact, $E$ is totally disconnected, or $E$ is a chain. We then obtain necessary and sufficient conditions in order that a given inverse system of compact groups indexed by a totally disconnected semilattice $E$ admit a compatible compact semigroup topology on their disjoint union.
\end{abstract}

It is the intent of this paper to present a result relating to the structure of the character semigroup of certain locally compact abelian inverse semigroups. A function $\chi$ defined on a (Hausdorff) topological semigroup $S$ is called a character of $S$ iff $\chi$ is a bounded continuous homomorphism from $S$ into the multiplicative semigroup $\boldsymbol{C}$ of complex numbers. We also require that if $S$ has an identity 1 , then $\chi(1) \neq 0$. Thus the function which is constantly zero on $S$ is a character iff $S$ does not have an identity. Note that $S$ actually has its values in the unit disc and in case $S$ is an inverse semigroup, $|\chi(s)|=1$ or $|\chi(s)|=0$ for each $s \in S$. The set of all characters of $S$ is a semigroup under pointwise multiplication of functions. Moreover, if the semigroup consisting of all of the characters of $S$ is topologized with the compact-open topology, it becomes a topological semigroup. We denote this topological semigroup of characters of $S$ by $S^{\wedge}$ and refer to it as the character semigroup of $S$.

Later in the paper we prove a theorem which determines the maximal subgroups of $S^{\wedge}$ in terms of the maximal subgroups of $S$ for certain locally compact continuous-inverse abelian semigroups. One corollary of that result is the following theorem.

THEOREM 1. Assume that $S$ is a locally compact abelian continuousinverse semigroup whose maximal idempotent subsemigroup $E$ either is

Presented to the Society, August 28, 1970; received by the editors December 23, 1969 and, in revised form, April 27, 1970.

AMS 1969 subject classifications. Primary 2205; Secondary 2092.

Key words and phrases. Locally compact abelian semigroups, character semigroups, inverse limits, duals of maximal groups, compact topologies, totally disconnected semilattices. 
a chain, is compact, or is totally disconnected. If, for each $e \in E, H_{e}$ is the maximal group of $S$ containing $e$ and $\lambda$ is an idempotent in $S^{\wedge}$, then the maximal subgroup of $S^{\wedge}$ containing $\lambda$ is iseomorphic to

$$
\operatorname{inv} \lim \left[\left\{H_{e}^{\hat{e}}\right\}_{\lambda(e) \neq 0} ;\left\{\pi_{f e}\right\}_{f \leq e}\right]
$$

wher, $\pi_{f e}: \hat{H_{f}} \rightarrow \hat{H_{e}}$ is defined by $\pi_{f e}(\chi)(s)=\chi(s f)$.

REMARK. In the notation of Theorem 1 , denotes the usual partial order defined on a commutative idempotent semigroup $E$ by requiring that $f \leqq e$ iff $e f=f$. However, in order to consider $\left\{\pi_{f e}\right\}_{f \leqq e}$ as an inverse system of maps it is necessary that the direction on the index set of the inverse system above be the dual partial order of the relation $\leqq$. With it understood that this is the case, we shall not require an additional notation for the direction on $\{e \in E \mid \lambda(e) \neq 0\}$. We also adhere to the convention that the inverse limit of a void family of groups is the trivial group.

We actually prove a theorem which appears to be stronger than Theorem 1 . The hypothesis on $E$ is relaxed by requiring only that $E$ satisfy a condition which shall be referred to as the separation hypothesis. If $E$ is a topological semilattice, we shall say that $E$ satisfies the separation hypothesis iff whenever $C \subseteq E$ is compact and $C \cap P=\varnothing$ for some clopen prime ideal $P$ of $E$, then there exists $e \in E$ such that $C \subseteq U(e)$ and $U(e) \cap P=\varnothing$ where $U(e)=\{g \in E \mid g \geqq e\}$. The set $U(e)$ will be called the upper set of $e \in E$ in $E$.

We first show that the separation hypothesis is implied by the various possible hypotheses on $E$ in Theorem 1 .

THEOREM 2. Assume that $E$ is a locally compact semilattice with identity. Then any one of the following conditions on $E$ is sufficient in order that $E$ satisfy the separation hypothesis:

(1) $E$ is compact.

(2) $E$ is totally disconnected.

(3) $E$ is a chain.

(4) Each clopen prime ideal $P$ of $E$ is the intersection of cocyclic clopen prime ideals of $E$ (an ideal $I$ of $E$ is cocyclic iff its complement is the upper set of some $e \in E)$.

Proof of Theorem 2. First observe that each clopen prime ideal of a compact semilattice is cocyclic, thus (1) implies (4). The fact that (1) implies the separation hypothesis will follow from the fact that (4) does.

To see that (2) implies the separation hypothesis, assume that $C$ is a compact subset of a totally disconnected locally compact semi- 
lattice $E$ and that $P$ is a clopen prime ideal of $E$ such that $C \cap P=\varnothing$. Since $E$ is locally compact, it is 0 -dimensional and thus we can cover $C$ with subsets of $E$ which are both compact and open and which do not intersect $P$. Since $C$ is compact there exists a finite number of such compact and open sets which cover $C$. It follows that it is no loss of generality to assume $C$ is both compact and open. Now if $e \in C$, then it follows from [2] that there exists a generating idempotent $e_{*} \in C$ such that $e \geqq e_{*}$ (this follows from the fact that generating idempotents are dense from below and $C$ is open). Thus $U\left(e_{*}\right)$ is closed and open and $C \subseteq \bigcup_{e \in C} U\left(e_{*}\right)$. There exists $e^{1}, e^{2}, \cdots, e^{n}$ in $C$ such that $C \subseteq \mathrm{U}_{i=1}^{n} U\left(e_{*}^{i}\right) \subseteq U(g)$ where $g=e_{*}^{1} \cdot e_{*}^{2} \cdots e_{*}^{n}$. Although we do not know that $g$ is in $C$, it is clear that $g \notin P$. Thus $U(g) \cap P=\varnothing$ and $C \subseteq U(g)$. The separation hypothesis follows.

We now show that if $E$ is a chain then the separation hypothesis holds. Recall that a compact partially ordered space always has a minimal element and thus any compact subset of the complement of a clopen prime ideal of a chain has a least element. The separation hypothesis follows immediately.

Finally we prove that the separation hypothesis follows from (4). Assume that $C \subseteq E$ is compact and that $P$ is a clopen prime ideal of $E$ such that $C \cap P=\varnothing$. Now there exists a family $F$ of clopen prime ideals each of which is cocylic such that $P=\bigcap_{Q \in F} Q$. Thus $C \subseteq E \backslash P$ $=\cup_{Q \in F}(E \backslash Q)$ and $C \subseteq\left(E \backslash Q_{1}\right) \cup \ldots \cup\left(E \backslash Q_{n}\right)$ for some $Q_{1}, Q_{2}, \ldots$, $Q_{n}$ in $F$. For $1 \leqq i \leqq n$, choose $e_{i} \in E$ such that $Q_{i}=\left\{x \in E \mid x \geq e_{i}\right\}$. Then $C \subseteq \bigcup_{i=1}^{n} U\left(e_{i}\right) \subseteq U\left(e_{1} \cdot e_{2} \cdots e_{n}\right)$. Since $P$ is prime, $e_{1} \cdot e_{2} \cdots e_{n}$ $\notin P$ and thus

$$
U\left(e_{1} \cdot e_{2} \cdots e_{n}\right) \cap P=\varnothing .
$$

The theorem follows.

Prior to stating our main theorem we have the following wellknown lemma.

Lemma. If $E=E(S)$ is the subsemigroup of all idempotents of a locally compact inverse semigroup $S$, then $E^{\wedge}$ is iseomorphic to the subsemigroup $E\left(S^{\wedge}\right)$ of all idempotents of $S^{\wedge}$.

Our main result is as follows.

THEOREM 3. Let $S$ denote a locally compact abelian continuous-inverse semigroup with maximal idempolent subsemigroup $E$ and maximal groups $\left\{H_{e}\right\}_{e \in E}$. If $E$ satisfies the separation hypothesis and $\lambda \in E\left(S^{\wedge}\right)$, then the maximal subgroup of $S^{\wedge}$ containing $\lambda$ is iseomorphic to

$$
\operatorname{inv} \lim \left[\left\{\hat{H_{e}}\right\}_{\lambda(e) \neq 0} ;\left\{\pi_{f_{e}}\right\}\right]
$$


where $\pi_{f e}: H_{f} \rightarrow H_{e}^{\hat{e}}$ is defined by $\pi_{f e}(\chi)(s)=\chi(s f)$. Thus

$$
\hat{S} \cong \underset{\lambda \in E^{\wedge}}{\bigcup} \operatorname{inv} \lim \left[\left\{\hat{H_{e}}\right\}_{\lambda(e) \neq 0} ;\left\{\pi_{f e}\right\}\right]
$$

Proof. For each $\lambda \in E\left(S^{\wedge}\right)$, let $S_{\lambda}$ denote the maximal subgroup of $S^{\wedge}$ containing $\lambda$ and let $H_{\lambda}$ denote the topological group inv $\lim \left[\left\{H_{e}^{\hat{e}}\right\}_{\lambda(e) \neq 0} ;\left\{\pi_{f e}\right\}\right]$. Let $\psi$ denote the function from $S_{\lambda}$ into

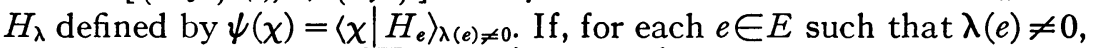
$\pi_{e}$ is the projection of $\prod_{\lambda(e) \neq 0} H_{e} \hat{~ o n t o ~} H_{e}^{\hat{e}}$ then it is clear that $\pi_{e} \circ \psi$ is continuous (recall that the topology on $S_{\lambda}$ is the relativization of the compact-open topology to $S_{\lambda}$ ). Thus $\psi$ is continuous and is clearly an injective homomorphism.

We now show that $\psi$ is surjective. Let $\left\{\chi_{e}\right\}_{\lambda(e) \neq 0} \in H_{\lambda}$. Define $\chi: S \rightarrow C$ by

$$
\begin{aligned}
& \chi(s)=\chi_{e}(s) \quad \text { if } s \in H_{e} \text { and } \quad \lambda(e) \neq 0 \text {, } \\
& =0 \quad \text { if } s \in H_{e} \text { and } \lambda(e)=0 .
\end{aligned}
$$

Since $\left\{\chi_{e}\right\}_{\lambda(e) \neq 0}$ is in $H_{\lambda}$ it is easy to see that $\chi$ is a homomorphism. We show that $\chi$ is continuous. Let $\left\{y_{\alpha}\right\}_{\alpha \in A}$ denote a net in $S$ with limit $y_{0} \in S$. If $\lambda\left(y_{0}\right)=0$ it follows that $\left\{\chi\left(y_{\alpha}\right)\right\}_{\alpha \in A}$ has limit $\chi\left(y_{0}\right)$ since $\{s \in S \mid \lambda(s)=0\}$ is open. Thus we assume that $y_{0} \in H_{e}$ for some $e \in E$ such that $\lambda(e) \neq 0$. There is an open set $U$ containing $y_{0}$ such that $\bar{U}$ is compact and $\bar{U} \subseteq\{s \mid \lambda(s) \neq 0\}$. The set $C$ $=\left\{e \in E \mid H_{e} \cap \bar{U} \neq \varnothing\right\}$ can be shown to be a compact subset of $E$ by means of a net argument and by use of the fact that $S$ is a continuousinverse semigroup. Clearly $C$ is disjoint from the clopen prime ideal $P=\{e \in E \mid \lambda(e)=0\}$ of $E$. By the separation hypothesis there exists $g \notin P$ such that $g \leqq f$ for each $f \in C$. Choose $\beta \in A$ such that $\alpha \geqq \beta$ implies $y_{\alpha} \in U$. Then $\left\{y_{\alpha} g\right\}_{\alpha \geq \beta}$ is a net in $H_{g}$ with limit $y_{0} g$ and thus $\left\{\chi_{\theta}\left(y_{\alpha} g\right)\right\}_{\alpha \geq \beta}$ has limit $\chi_{\theta}\left(y_{0} g\right)$. It follows that $\left\{\chi\left(y_{\alpha}\right)\right\}_{\alpha \in A}$ has limit $\chi\left(y_{0}\right)$ and that $\chi$ is continuous. Thus $\chi \in H_{\lambda}$ and $\psi(\chi)=\left\{\chi_{e}\right\}_{\lambda(e) \neq 0}$.

We now show that $\psi$ is an open map. It suffices to show that if $U$ is open about the identity $\lambda$ of $S_{\lambda}$, then there exists $V$ open about the identity in $\prod_{\lambda(e) \neq 0} H_{e}^{\hat{e}}$ such that $V \cap \psi\left(S_{\lambda}\right) \subseteq \psi(U)$ (see Hewitt and Ross $\left[4\right.$, p. 50]). If $U$ is open about $\lambda$ in $S_{\lambda}$, then there exists a compact subset $K$ of $S$ and an open set $\Lambda$ about the identity 1 in the circle group such that $\lambda \in[K, \Lambda \cup\{0\}] \cap S_{\lambda}$ and $[K, \Lambda \cup\{0\}] \cap S_{\lambda}$ $\subseteq U$ (if $A \subseteq S$ and $B \subseteq C$, then $[A, B]$ denotes the set of all $\chi \in S^{\wedge}$ such that $\chi(A) \subseteq B)$. Let $s_{\lambda}$ denote the complement of the clopen prime ideal $\{x \in S \mid \lambda(x)=0\}$ of $S$ and let $C=\left\{e \in E \mid H_{e} \cap s_{\lambda} \cap K \neq \varnothing\right\}$. Then $C$ is a compact subset of $E$ disjoint from the prime ideal 
$E \backslash\left(E \cap s_{\lambda}\right)$ of $E$. By our separation hypothesis there exists $g \in E \cap s_{\lambda}$ such that $g \leqq e$ for each $e \in C$. Let $K^{*}=K \cap s_{\lambda}$, then $K^{*} \subseteq \bigcup_{o s e} H_{e}$ and $g K^{*} \subseteq H_{g}$. If $\left[g K^{*}, \Lambda\right]_{g}$ denotes all those members of $H_{\lambda}$ which send $g K^{*}$ into $\Lambda$, then $\left[g K^{*}, \Lambda\right]_{g}$ is open in $H_{\sigma}^{\hat{\sigma}}$ and $V=\pi_{g}^{-1}\left(\left[g K^{*}, \Lambda\right]_{g}\right)$ is open in $\prod_{\lambda(e) \neq 0} H_{e}^{\hat{e}}$. We show that $V \cap \psi\left(S_{\lambda}\right) \subseteq \psi(U)$. Let $\chi \in S_{\lambda}$ such that $\psi(\chi) \in V$. Then $\chi \mid H_{o}=\pi_{g}(\psi(\chi))$ belongs to $\left[g K^{*}, \Lambda\right]_{\theta}$ and $\chi\left(g K^{*}\right) \subseteq \Lambda$. Thus $\chi\left(K \cap s_{\lambda}\right) \subseteq \Lambda$ and $\chi(K) \subseteq \Lambda \cup\{0\}$. It is clear that $\chi \in U$. It follows that $\psi$ is an open mapping and consequently is an iseomorphism.

Corollary. Assume that $S$ is a locally compact abelian continuousinverse semigroup. If each clopen prime ideal of $E(S)$ is cocyclic, then the maximal subgroup of $S^{\wedge}$ containing $\lambda \in E\left(S^{\wedge}\right)$ is iseomorphic to $H_{e(\lambda)}$ where $e(\lambda)$ is the least element of $\{e \in E \mid \lambda(e) \neq 0\}$. Thus $S^{\wedge}$ $=\bigcup_{\lambda \in E^{\wedge}} \hat{H_{e(\lambda)}}$.

Proof. Under the hypothesis of the corollary we clearly have Theorem 3, thus for $\lambda \in E\left(S^{\wedge}\right)$, the maximal group of $\lambda$ is inv $\lim \left[\left\{\hat{H_{e}}\right\}_{\lambda(e) \neq 0} ;\left\{\pi_{f e}\right\}\right]$. But if $e(\lambda)$ is the least $e$ such that $\lambda(e)$ $\neq 0$, then $e(\lambda)$ is the largest element in the direction of the inverse system of groups above (recall the remark following Theorem 1) and thus the inverse limit is iseomorphic to $\hat{H_{e(\lambda)}}$. The corollary follows.

REMARK. It is now clear that Theorem 1 holds as it is an immediate consequence of Theorems 2 and 3.

We now state a corollary of our results which will be of some use in a subsequent publication. Its proof follows from Theorem 3 and the results of [1] and [2].

Let $\left\{S_{e}\right\}_{e \in E}$ denote a family of compact topological groups indexed by a compact totally disconnected semilattice $E$ with identity. For $f \leqq e$ in $E$ assume that $\pi_{f e}: S_{e} \rightarrow S_{f}$ is a continuous homomorphism. Define a semigroup structure on the disjoint union $\bigcup_{e \in E} S_{e}$ by $a b$ $=\pi_{e f, e}(a) \pi_{e f, f}(b)$ for $a \in S_{e}$ and $b \in S_{f}$. We seek conditions under which there exists a compact topology on $\bigcup_{e \in E} S_{e}$ which relativizes to the given topology on $E$ and on the various $S_{e}$ and with respect to which $\bigcup_{e \in E} S_{e}$ is a compact semigroup. For each $e \in E$, let $E(e)$ denote the set of generating idempotents of $E$ which lie in $e E$ (recall that an idempotent $f$ of $E$ is a generating idempotent iff the prime ideal of $E$ determined by $f$ is clopen). In [2] it was shown that, for each $e \in E$, $E(e)$ is nonvoid and that actually there exists a net in $E(e)$ which converges upward to $e$. Consider the map

$$
\pi: S_{e} \rightarrow \operatorname{inv} \lim \left[\left\{S_{f}\right\}_{f \in E(e)} ;\left\{\pi_{f^{\prime} f}\right\}_{f^{\prime} \leq f}\right]
$$


defined by $\pi(x)=\left\langle\pi_{f e}(x)\right\rangle_{f s e}$. This function is always a continuous homomorphism. We have the theorem.

THEOREM 4. The semigroup $\bigcup_{e \in E} S_{e}$ admits a compact semigroup topology which relativizes to the given topologies on $E$ and on $S_{e}$, for each $e \in E$, iff the mapping $\pi$ is an iseomorphism from $S_{e}$ onto inv $\lim \left[\left\{S_{f}\right\}_{f \in E(e)} ;\left\{\pi_{f^{\prime} f}\right\}\right]$. Moreover, whenever such a topology exists it is unique.

We very briefly indicate the proof that if $\pi$ is an iseomorphism then $\bigcup_{e \in E} S_{e}$ admits a compact topology. Let $T$ denote the disjoint union $U_{\lambda \in E(S) \wedge} S_{e(\lambda)}^{\wedge}$ where $e(\lambda)$ is the least $e \in E$ such that $\lambda(e) \neq 0$. Define a semigroup structure on $T$ via the naturally induced system of mappings. Now by [1], $T^{\wedge}$ is a compact topological semigroup ( $T$ is discrete). One can show that $T^{\wedge}$ is algebraically isomorphic to $\bigcup_{e \in E} S_{e}$ by showing that the maximal groups of $T^{\wedge}$ are the various $S_{e}$ for $e \in E$. This latter statement is proven via Theorem 1, the iseomorphism $E \cong E^{\wedge \wedge}$ (see [2]), and our hypothesis which insures that

$$
S_{e} \cong \operatorname{inv} \lim \left\{S_{f}\right\}_{f \in E(e)} .
$$

All other assertions of Theorem 4 are proven in [2].

Finally the author wishes to acknowledge the numerous helpful suggestions of the referee. Among these is the improved proof that (4) implies the separation hypothesis of Theorem 2.

\section{REFERENCES}

1. C. W. Austin, Duality theorems for some commutative semigroups, Trans. Amer. Math. Soc. 109 (1963), 245-256. MR 27 \#3737.

2. J. W. Baker and N. J. Rothman, Separating points by semicharacters in topological semigroups, Proc. Amer. Math. Soc. 21 (1969), 235-239. MR 38 \#4598.

3. A. H. Clifford and G. B. Preston, Algebraic theory of semigroups. Vol. I, Math. Surveys, no. 7, Amer. Math. Soc., Providence, R. I., 1961. MR 24 \#A2627.

4. E. Hewitt and K. A. Ross, Abstract harmonic analysis. Vol. I: Structure of topological groups. Integration theory, group representation, Die Grundlehren der math. Wissenschaften, Band 115, Academic Press, New York; Springer-Verlag, Berlin, 1963. MR $28 \# 158$.

5. S. Schwarz, The theory of characters of commutative Hausdorff bicompact semigroups, Czechoslovak Math. J. 6 (81) (1956), 330-364. MR 19, 1063.

North Carolina State University, Raleigh, North Carolina 27607 